\title{
Expression of CD147 and matrix metalloproteinase-11 in colorectal cancer and their relationship to clinicopathological features
}

Xiuyun Tian ${ }^{1 \dagger}$, Chunxiang Ye ${ }^{1,2+}$, Yongyong Yang ${ }^{3}$, Xiaoya Guan ${ }^{1}$, Bin Dong ${ }^{1}$, Min Zhao ${ }^{1}$ and Chunyi Hao ${ }^{1 *}$

\begin{abstract}
Background: This study aimed to investigate the expression of CD147 and MMP-11 in human colorectal cancer $(C R C)$ and to evaluate their clinical significance.

Methods: Real-time polymerase chain reaction was used to evaluate CD147 and MMP-11 mRNA level in 56 pairs of fresh CRC samples matched with adjacent normal mucosa. The protein expression of CD147 and MMP-11 in CRC specimens and corresponding normal colorectal mucosa were evaluated by immunohistochemistry on CRC tissue microarrays. Expression and co-localization of these two proteins in human colorectal cancer tissue were also evaluated by laser scanning confocal microscopy. Furthermore, their correlations with clinicopathological factors and overall survival after surgery were evaluated.

Results: Both CD147 and MMP-11 were demonstrated to be over-expressed at mRNA level $(P<0.001$, both) and protein level $(P<0.001$, both) in CRC tissue than paired normal mucosa. Spearman rank test showed a positive correlation between these two proteins $(P=0.025)$. Immunofluorescence double staining confirmed the co-localization of CD147 and MMP-11 in paraffin-embedded tissues of CRC patients. Expression of CD147 and MMP-11 were both correlated with CRC lymph node metastasis $(P=0.021$ and $P=0.031$, respectively), distant metastasis $(P<0.001$ and $P=0.013$, respectively) and TNM stage $(P=0.006$ and $P=0.049$, respectively). Univariate survival analysis showed that both CD147 and MMP11 expression was significantly associated with shorter survival time $(P=0.001$ and $P=0.009$, respectively). Additionally, in multivariate analysis, both CD147 and MMP-11 were proved to be independent prognostic factors ( $P=0.009,0.028$, respectively).
\end{abstract}

Conclusions: These results indicated that both CD147 and MMP-11 may be involved in the progression of colorectal cancer, and they are potential prognostic factors and might become new therapeutic targets for CRC patients.

Keywords: Colorectal cancer, Immunohistochemistry, CD147, MMP-11, Prognosis

\section{Background}

Colorectal cancer is one of the most prevalent malignant tumors and a main cause of cancer related death

\footnotetext{
*Correspondence: haochunyi@bjmu.edu.cn

${ }^{+}$Xiuyun Tian and Chunxiang Ye contributed equally to this work

1 Key Laboratory of Carcinogenesis and Translational Research,

Ministry of Education/Beijing, Department of Hepato-Pancreato-Biliary

Surgery, Peking University Cancer Hospital and Institute, Beijing, People's

Republic of China

Full list of author information is available at the end of the article
}

worldwide. Its incidence has been increasing in China in recent years. Although most patients at early stage can be successfully cured with surgery, about 20-45\% of patients who underwent curative resection developed recurrence [1]. What's more, about $15-25 \%$ of all CRC patients have a synchronous liver metastasis, of which $80-90 \%$ are unresectable [2,3].Elucidation of the malignant biological behavior, especially the metastasis mechanism, is expected to bring more benefit to the CRC patients in the long run. 
Matrix metalloproteinases (MMPs) are a family of zincdependent endopeptidases that have been extensively studied during the past years, and a variety of studies have established their crucial roles in the invasion and metastasis process of malignant tumors through degradation of the extracellular matrix (ECM) and some other mechanisms. MMP-11 (also known as stromelysin-3) is a member of the MMPs family. It was originally identified as a highly expressed protein in the stromal cells of invasive breast cancer compared to that of fibroadenoma as determined by subtractive hybridization [4]. Unlike other MMPs, MMP-11 has some unique properties. While most MMPs are secreted as inactive zymogens, MMP-11 is released as a $45 \mathrm{kDa}$ active enzyme [5]. In particular, MMP-11 does not appear to degrade any classical extracellular matrix component, instead, it catalyzes the degradation of serine protease inhibitors, a 1-antitrypsin and insulin-like growth factor binding protein-1 (IGF-BP-1) $[6,7]$, and it may probably only play an indirect role in ECM remodeling, which is different from other MMPs. These unique properties indicate that MMP-11 might play some unique roles in malignant tumor development and progression. Previous studies have investigated its role in breast cancer $[8,9]$ and gastric cancer [10], while its role in colorectal cancer has rarely been investigated.

The expression and activity of MMPs are regulated under a complex mechanism, which involves a lot of signal pathways and related molecules [11], of which CD147 plays a key role. CD147, also known as extracellular matrix metalloproteinase inducer (EMMPRIN), is a member of the immunoglobulin superfamily of adhesion molecules. It plays an essential role in tumor progression and metastasis through stimulating tumor cells to secrete matrix metalloproteinases (MMPs). The relationship between CD147 and MMP-9, MMP-2 and their relevance with patients' prognosis and clinicopathological parameters have been studied in a variety of cancers. However, the correlation between CD147 and MMP-11 expression has rarely been investigated until now.

In the present study, we investigated CD147 and MMP11 expression in colorectal cancer. We aimed to make it clear the expression level of these two proteins in CRC tissue compared with normal mucosa, and their relationship with the clinicopathological parameters and prognosis of the CRC patients, which could provide more information for elucidating the molecular mechanism and target therapy of the disease.

\section{Methods}

\section{Patients and samples}

A total of 218 CRC patients who were diagnosed and underwent surgery in Peking University Cancer Hospital between 2006 and 2009 were included in this study.
Fresh colorectal carcinoma specimens and paired adjacent normal mucosa from each patient were formalin-fixed, paraffin-embedded, and constructed into tissue microarrays. Postoperative follow-up has lasted at least 3 years for all of these patients. Patients who received chemotherapy or radiation therapy before surgery were excluded. Histopathological evaluation was carried out independently by two pathologists. This study was approved by the institutional review board of Peking University Cancer Hospital, and written informed consent was acquired from each patient. The characteristics of all the patients are shown in Table 1.

\section{Quantitative real-time PCR}

Total RNA was extracted from 56 pairs of fresh tissues using Trizol reagent (Life Technologies), according to the manufacturer's instructions, and the RNA concentration was determined using an Ultrospec ${ }^{\circledR} 3300$ Pro (GE Health Care, Little Chalfont, Buckinghamshire, UK). Reverse transcription was performed using $4000 \mathrm{ng}$ of total RNA in a $20 \mu \mathrm{L}$ reaction volume using the EasyScript FirstStrand cDNA Synthesis SuperMix Kit (Life Technologies). The mRNA expression of CD147 and MMP-11 was examined by real-time PCR using Power SYBR ${ }^{\circledR}$ Green PCR Master Mix (Life Technologies) with gene-specific primers and the ABI 7500 Real-time PCR Detection System. The primer sequences were as follows: CD147: 5'-GGCTGTGAAGTCGTCAGAACAC-3' (sense) and 5'-ACCTGCTCTCGGAGCCGTTCA-3' (antisense); MMP-11: 5'-GAGAAGACGGACCTCACCTACA-3' (sense) and 5'-CTCAGTAAAGGTGAGTGGCGTC-3' (antisense); beta-actin: 5'-TTAGTTGCGTTACAC CCT TTC-3' (sense) and 5'-ACCTTCACCAGTTC CAGTTT- $3^{\prime}$ (antisense). The thermal cycling conditions involved predenaturation at $95^{\circ} \mathrm{C}$ for $10 \mathrm{~min}$, followed by 40 cycles of denaturation at $95{ }^{\circ} \mathrm{C}$ for $15 \mathrm{~s}$, annealing at $60{ }^{\circ} \mathrm{C}$ for $1 \mathrm{~min}$, and extension at $72{ }^{\circ} \mathrm{C}$ for $30 \mathrm{~s}$. All reactions were performed in triplicate. The mRNA expression was measured using threshold cycle values $\left(C_{t}\right)$. To normalize this value, a $\Delta \mathrm{C}_{\mathrm{t}}$ value was determined as the difference between the $C_{t}$ value for each gene and the $C_{t}$ value for $\beta$-actin. Then, a $\Delta \Delta C_{t}$ value was determined as the difference between the $\Delta C_{t}$ value for each individual sample and the average $\Delta C_{t}$ value for this gene, obtained from the control samples. The results were presented as fold changes, calculated using the $2^{-\Delta \Delta C t}$ method (Livak and Schmittgen 2001). The comparative $C_{t}$ method, normalizing $C_{t}$ values to $\beta$-actin, was used to calculate the relative expression level of CD147 and MMP-11.

\section{Colorectal cancer tissue microarray (TMA) construction}

All specimens were stained with hematoxylin and erosin, and matched representative cancerous and adjacent 
Table 1 Summary of patient characteristics $(n=218)$

\begin{tabular}{|c|c|c|}
\hline Clinicopathological features & No. of patients & (\%) of patients \\
\hline \multicolumn{3}{|l|}{ Gender } \\
\hline Male & 129 & 59.17 \\
\hline Female & 89 & 40.83 \\
\hline \multicolumn{3}{|l|}{ Age } \\
\hline$<60$ & 99 & 45.41 \\
\hline$\geq 60$ & 119 & 54.59 \\
\hline \multicolumn{3}{|l|}{ Tumor size (cm) } \\
\hline$<4$ & 104 & 47.71 \\
\hline$\geq 4$ & 114 & 52.29 \\
\hline \multicolumn{3}{|l|}{ Lymphovascular invasion } \\
\hline Absent & 149 & 68.35 \\
\hline Present & 69 & 31.65 \\
\hline \multicolumn{3}{|l|}{ Differentiation } \\
\hline Well-moderate & 179 & 82.11 \\
\hline Poor & 39 & 17.89 \\
\hline \multicolumn{3}{|l|}{ Depth of invasion } \\
\hline $\mathrm{T} 1+\mathrm{T} 2$ & 33 & 15.14 \\
\hline $\mathrm{T} 3+\mathrm{T} 4$ & 185 & 84.86 \\
\hline \multicolumn{3}{|l|}{ Lymph node metastasis } \\
\hline NO & 89 & 40.83 \\
\hline$N 1-2$ & 129 & 59.17 \\
\hline \multicolumn{3}{|l|}{ Liver metastasis } \\
\hline Mo & 106 & 48.62 \\
\hline M1 & 112 & 51.38 \\
\hline \multicolumn{3}{|l|}{ TNM stage } \\
\hline $1+\|$ & 75 & 34.40 \\
\hline$I I I+I V$ & 143 & 65.60 \\
\hline
\end{tabular}

normal mucosa from paraffin blocks were isolated from 218 colorectal cancer patients. For each patient, three cylinders (the diameter is $1.0 \mathrm{~mm}$ ) of representative cancerous paraffin blocks and two cylinders (the diameter is $1.0 \mathrm{~mm}$ ) of normal mucosa paraffin blocks were obtained. To obtain a broad coverage of the tumor tissue, the three regions were preferentially chosen from the tumor center and the invasive margins. Then, the representative cylinders were arrayed into a receptor paraffin block by an automated tissue-arraying instrument (ALPHELYS) and reheated for $30 \mathrm{~min}$ at $37^{\circ} \mathrm{C}$. The $4 \mu \mathrm{m}$ sections were then cut from the resulting TMA block.

\section{Immunohistochemistry assay}

TMA block sections $\left(4 \mu \mathrm{m}\right.$ thick) were baked at $70{ }^{\circ} \mathrm{C}$, dewaxed with xylene, and rehydrated with graded alcohol washes. Antigen retrieval was performed in a pressure cooker, followed by the treatment with $3 \%$ hydrogen peroxide for 15 min to block endogenous peroxidase activity. Thereafter, the sections were incubated at $4{ }^{\circ} \mathrm{C}$ overnight with anti-CD147 (ab78106, abcam) or
anti-MMP-11(ab52904, abcam). The stained specimens were exposed to the 3,3-diam-inobenzidine (DAB) Kit (Zhong shan Biotechnology Inc., Beijing, China) and counterstained with hematoxylin. For the negative controls, primary antibodies were replaced with PBS.

\section{Immunofluorescence assay}

Double-fluorescence staining of CD147 and MMP-11 were conducted on formalin-fixed, paraffin-embedded tissue sections from 20 CRC patients. The slides were baked at $70^{\circ} \mathrm{C}$, dewaxed with xylene, and rehydrated with graded alcohol washes. Antigen retrieval was performed in a pressure cooker, followed by the treatment with $3 \%$ hydrogen peroxide for 15 min to block endogenous peroxidase activity. Thereafter, the sections were incubated at $4{ }^{\circ} \mathrm{C}$ overnight with anti-CD147 (ab78106, abcam) or anti-MMP-11(ab52904, abcam). Then the CD147 and MMP-11 primary antibodies were detected by secondary antibodies as follows: Goat anti-mouse IgG (Zhong shan Biotechnology Inc., Beijing, China, ZF-0312, green) and Goat anti-rabbit IgG (Zhong shan Biotechnology Inc., Beijing, China, ZF-0316, red). After washing for two times, nuclei were stained with $4^{\prime}, 6^{\prime}$ - diamidino-2-phenylindole (DAPI, Zhong shan Biotechnology Inc., Beijing, China, ZLI-9557) at room temperature for $10 \mathrm{~min}$, and stored at $4{ }^{\circ} \mathrm{C}$. The slides were examined using a laser scanning confocal microscope (Zeiss LSM510). Images were collected and processed using the Zeiss AIM software and sized in Adobe Photoshop CS6.

\section{Evaluation immunohistochemical of staining}

The CD147 and MMP-11 staining were microscopically examined and scored by two independent pathologists who were blind to the clinical data pertaining to the patients. For CD147 and MMP-11 immunohistochemical staining assessment, immunoreactivity score (IRS) was assessed which evaluated both the percentage of positive cells and the staining intensity. The percentage of positive cells was scored as 0 (negative), 1 ( $<25 \%), 2$ (25-75\%), and 3 (>75\%); staining intensity was graded as 0 (colorless), 1 (pallide-flavens), 2 (yellow), and 3 (brown). The above two scores were multiplied, and "negative" and "positive" expression were defined according to IRS values of 0 and $>0$, respectively.

\section{Statistical analysis}

Statistical analysis was carried out using SPSS software package version 16.0 (SPSS Inc., Chicago, IL, USA). The differential expression of CD147 and MMP-11 mRNA between colorectal cancer tissues and adjacent normal mucosa was compared using a nonparametric test. Two-tailed $X^{2}$ test was used to evaluate the expression difference between colorectal cancer tissue and normal 
mucosa, as well as the relationship between the clinicopathological features and CD147 or MMP-11 expression. Correlation between CD147 and MMP-11was evaluated using the Spearman rank test. The survival curves were estimated by Kaplan-Meier analysis, and $P$ values were calculated by log rank test. The effect of different factors on patient survival was performed by multivariate analysis with the Cox proportional hazards regression model. $P<0.05$ was considered significant.

\section{Results}

mRNA expression of CD147 and MMP-11 in fresh, paired

\section{CRC tissues}

Both CD147 and MMP-11 mRNA expression levels in 56 pairs of primary CRC and matched normal mucosa were examined using real-time PCR. Results showed that the mRNA levels of both CD147 and MMP-11 were much higher in tumor tissues than in normal tissues $(P<0.001$, both) (Fig. 1).

\section{CD147 and MMP-11 expression in CRC tissues by IHC} and immunofluorescence double staining

Immunohistochemical staining showed a membranous and cytoplasmic staining of both CD147 and MMP-11 of tumor cells (Fig. 2). CD147 expression in normal mucosa was significantly lower than that in colorectal cancer (9.17 vs. $59.63 \%, P<0.001)$. Similarly, there was also a significantly higher expression of MMP-11 in colorectal cancer tissue than in normal mucosa (77.06 vs. $33.94 \%$, $P<0.001$ ) (see Table 2). Spearman's rank correlation coefficient test showed a positive correlation between CD147 and MMP-11 expression in CRC tissues $\left(r_{s}=0.152\right.$, $P=0.025)$. Immunofluorescence double staining assay confirmed the intracellular localization of CD147 and MMP-11 shown in immunohistochemical staining, and evident co-localization can be observed between these two proteins (Fig. 3).

Association of immunohistochemical expression of CD147 and MMP-11 with clinicopathological features

Based on staining evaluation and the statistical analysis, we further investigated the correlation between CD147/MMP-11 expression and the clinicopathological features as shown in Table 3. CD147 expression was markedly higher in patients with lymph node metastasis $(P=0.021)$, distant metastasis $(P<0.001)$, and advanced TNM stage $(P=0.006)$. Interestingly, MMP-11 expression was also significantly correlated with the above three parameters, with $P=0.031,0.013,0.049$, respectively. However, both CD147 and MMP-11 expression was not correlated with patient's gender, age, differentiation, tumor size, and depth of invasion. Detailed results are shown in Table 3.

\section{Prognostic implication of CD147 and MMP-11 in colorectal cancer}

The follow-up time of all patients was from February 2006 to July 2014. The overall median survival time was 49.35 months. During the follow-up period, a total of 79 patients died of colorectal cancer. The survival curves were estimated by Kaplan-Meier analysis and then compared using the log-rank test. Results showed that patients with CD147 or MMP-11 expression had a poorer prognosis $(P=0.001$ and 0.009 , respectively) (Fig. 4$)$. To test the prognostic value of combined expression status of CD147 and MMP-11, we classified all patients into two groups, CD147+/MMP-11+ group and all other expression statuses as the other group. Kaplan-Meier analysis showed that patients with CD147+/MMP-11+ had a significantly poor survival time than those with other
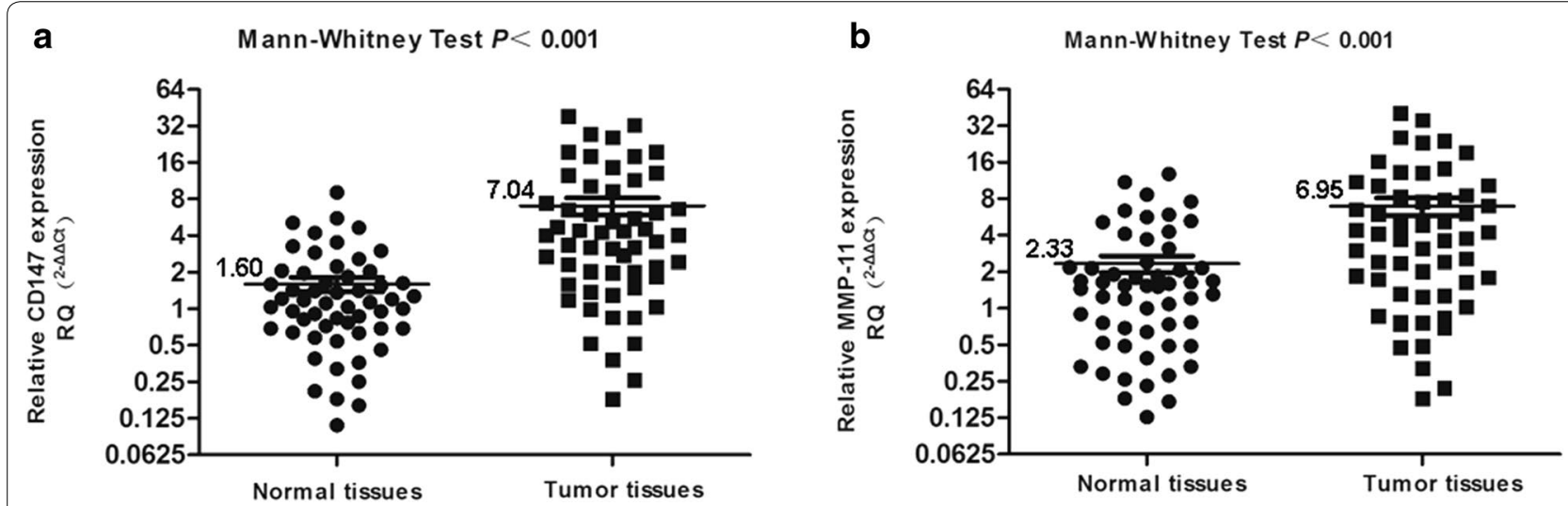

Fig. 1 Real-time PCR evaluation of CD147 and MMP-11 mRNA expression in 14 pairs of CRC tissues and matched normal mucosa. a mRNA expression of CD147 in tumor tissues was higher than that in normal tissues $(P=0.002)$. b mRNA expression of MMP-11 in tumor tissues was higher than that in normal tissues, too $(P=0.043)$. The relative quantification $(R Q)$ was calculated using the $2^{-\Delta \Delta c t}$ method 

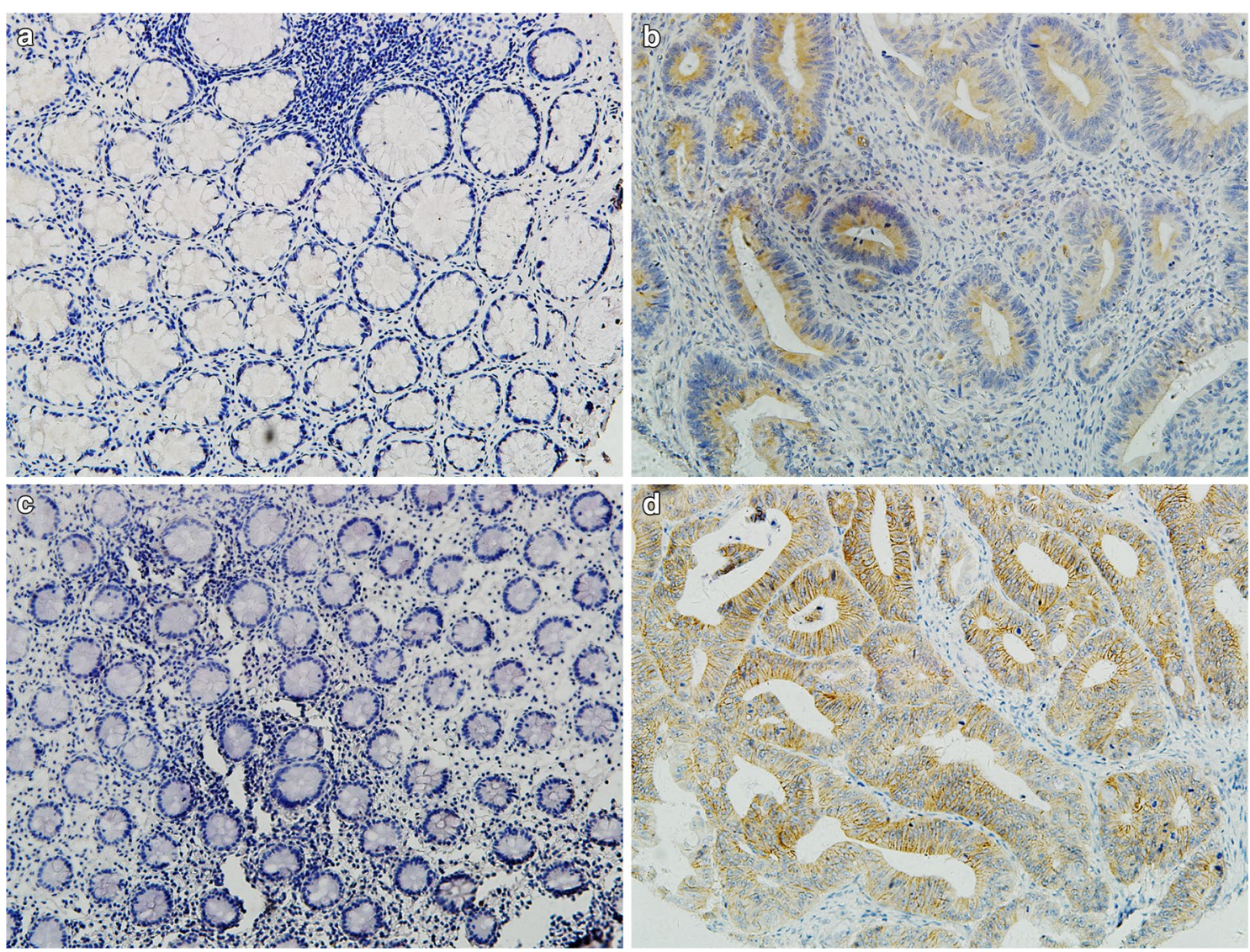

Fig. 2 Representative immunohistochemical staining of CD147 and MMP-11 in CRC tissue and matched normal mucosa. a, b show CD147 staining in normal mucosa and CRC tissue, respectively (magnification $\times 100$ ). $\mathbf{c}$, $\mathbf{d}$ show MMP-11 staining in normal mucosa and CRC tissue, respectively (magnification $\times 100$ ). As is shown, CD147 protein is predominantly localized on cell membrane and in the cytoplasm, while MMP-11 protein was of cytoplasmic staining

Table 2 Expression of CD147 and MMP-11 in carcinoma tissue and matched normal mucosa of CRC patients

\begin{tabular}{|c|c|c|c|c|c|c|}
\hline \multirow[t]{2}{*}{ Groups } & \multicolumn{3}{|c|}{ CD147 expression } & \multicolumn{3}{|c|}{ MMP-11 expression } \\
\hline & Positive (\%) & Negative (\%) & $P$ value & Positive (\%) & Negative (\%) & $P$ value \\
\hline Carcinoma tissue & $135(61.93)$ & $83(38.07)$ & $<0.001$ & $168(77.06)$ & $50(22.94)$ & $<0.001$ \\
\hline Normal mucosa & $21(9.63)$ & $197(90.37)$ & & $74(33.94)$ & $144(66.06)$ & \\
\hline
\end{tabular}

expression statuses $(P=0.002)$. Then, to test whether CD147 and MMP-11 expression were independent prognostic factors for CRC patients, we performed a multivariate survival analysis, in which those parameters associated with overall survival in the univariate survival analysis were included. In univariate analysis, CD147 expression $(P=0.001)$, MMP-11 expression $(P=0.011)$, differentiation $(P<0.001)$, lymphovascular invasion $(P<0.001)$, and tumor staging $(P<0.001)$ were associated with overall survival. In multivariate analysis lymphovascular invasion, CD147, MMP11 and tumor staging were independent prognostic factors related to overall survival ( $P=0.044,0.009,0.028,<0.001$, respectively). Detailed data are shown in Table 4.

\section{Discussion}

Local invasion and distant metastasis are suggested to be the key reasons for poor prognosis and cancer related death in tumor patients. Both these two proteins investigated in this study are closely related with tumor invasion and metastasis. In this study we found that there was an increased expression of CD147 and MMP-11 in 

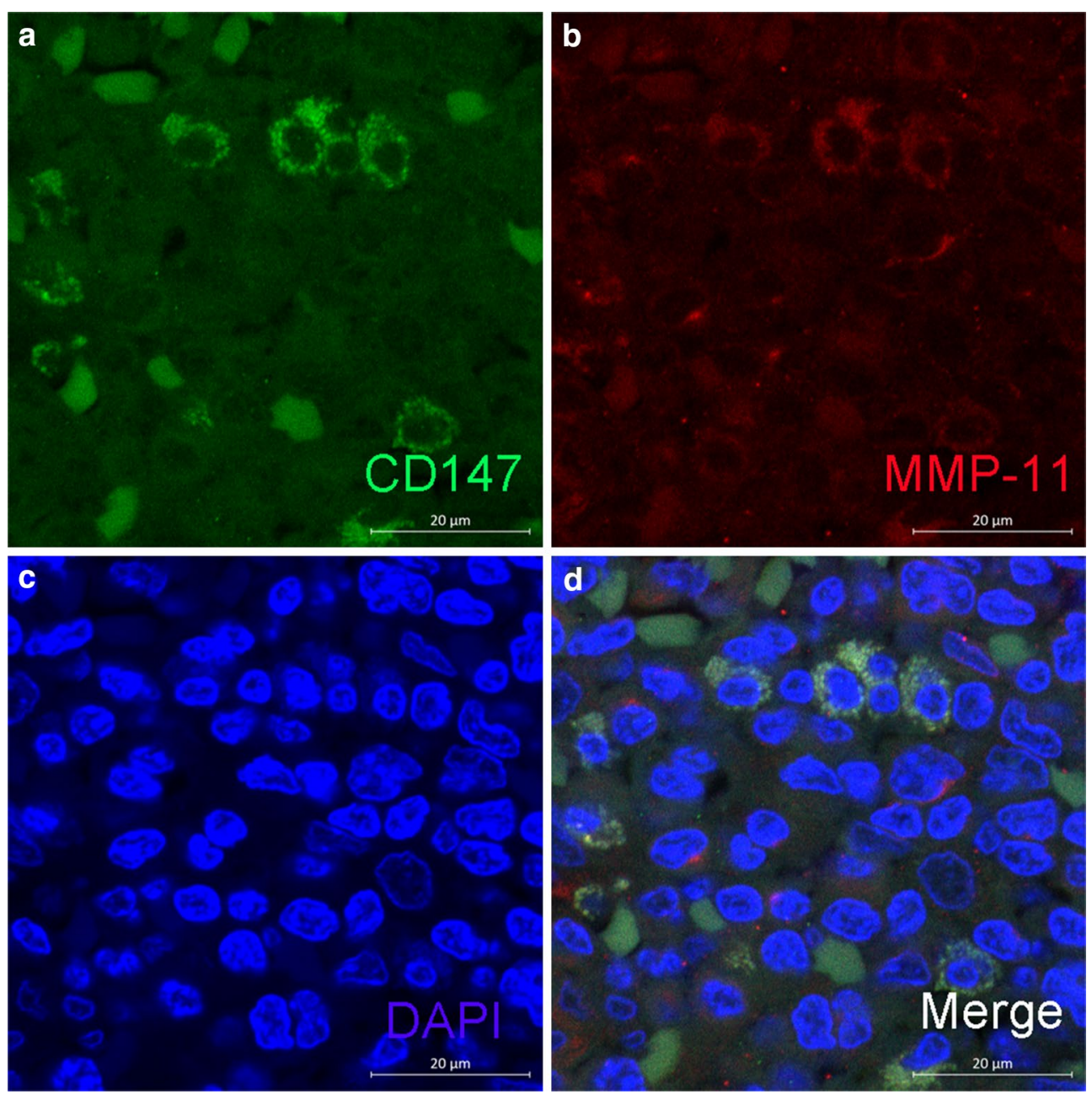

Fig. 3 Representative photomicrographs of double fluorescent staining of CD147 with MMP-11. Both a CD147 (green) and b MMP-11 (red) showed a predominant membranous and cytoplasmic localization. c DAPI (blue) was stained to identify nuclei. d Co-localization of CD147 and MMP-11 (yellow) is apparent. Scale bar $20 \mu \mathrm{m}$

colorectal cancer compared with paired normal mucosa, suggesting that CD147 and MMP-11 might play an oncogenic role in colorectal cancer.

It has been well documented that CD147 induces the expression of several MMPs, such as MMP-1, MMP-2, MMP-9 [12], which are associated with tumor migration and invasion. The mechanisms by which CD147 up-regulates MMPs family appear to depend on its stimulation of the tumor-associated fibroblasts. The expression association between CD147 and MMP-2, MMP-9 has been well established in a series of human malignant tumors, such as gallbladder carcinoma, thyroid carcinoma, breast cancer, gastric cancer and colorectal cancer. While up to now, reports on the relationship between CD147 and MMP-11 are limited. Jia et al. reported that in murine hepatocarcinoma Hca-F cells, depletion or deglycosylation of CD147 down-regulated MMP-11 expression [13]. In another study, knockdown of CD147 reduced the secretion of MMP-11 in nasopharyngeal carcinoma [14]. In our study, we explored the relationship between CD147 and MMP11 expression in colorectal cancer, and found a positive correlation between these two proteins, and immunofluorescence double staining showed co-localization of these two proteins, which has provided new evidence for the regulatory interaction between CD147 and MMP-11.

Recent studies have established a role of oncogenic and tumor promoter for CD147, and its expression has been proved to be related with clinicopathological characteristics in a variety of cancer types. Zhong et al. [15] found that CD147 expression was correlated with higher incidence of lymph node metastasis and lower differentiation in stage T1 pulmonary adenocarcinoma; Zhao et al. [16] found 
Table 3 Association of CD147 and MMP-11 expression with clinicopathological features

\begin{tabular}{|c|c|c|c|c|c|c|}
\hline \multirow[t]{2}{*}{ Clinicopathological features } & \multicolumn{3}{|c|}{ CD147 expression } & \multicolumn{3}{|c|}{ MMP-11 expression } \\
\hline & Positive (\%) & Negative (\%) & $P$ value & Positive (\%) & Negative (\%) & $P$ value \\
\hline \multicolumn{7}{|l|}{ Gender } \\
\hline Male & $83(64.34)$ & $46(35.66)$ & 0.377 & $99(76.74)$ & $30(23.26)$ & 0.892 \\
\hline Female & $52(58.43)$ & $37(41.57)$ & & $69(77.53)$ & $20(22.47)$ & \\
\hline \multicolumn{7}{|l|}{ Age (years) } \\
\hline$<60$ & $57(57.58)$ & $42(42.42)$ & 0.228 & $77(77.78)$ & $22(22.22)$ & 0.819 \\
\hline$\geq 60$ & $78(65.53)$ & $41(34.45)$ & & $91(76.47)$ & $28(23.53)$ & \\
\hline \multicolumn{7}{|l|}{ Differentiation } \\
\hline Poor & $26(66.67)$ & $13(33.33)$ & 0.501 & $32(82.05)$ & $7(17.95)$ & 0.414 \\
\hline Moderate-well & 109 (60.89) & $70(39.11)$ & & $136(75.98)$ & $43(24.02)$ & \\
\hline \multicolumn{7}{|l|}{ Size } \\
\hline$<4 \mathrm{~cm}$ & $62(59.62)$ & $42(40.38)$ & 0.502 & $81(77.88)$ & $23(22.12)$ & 0.783 \\
\hline$\geq 4 \mathrm{~cm}$ & $73(64.04)$ & $41(35.96)$ & & $87(76.32)$ & $27(23.68)$ & \\
\hline \multicolumn{7}{|l|}{ Lymphovascular invasion } \\
\hline Absent & $93(62.42)$ & $56(37.58)$ & 0.827 & $110(73.83)$ & $39(26.17)$ & 0.095 \\
\hline Present & $42(60.87)$ & $27(39.13)$ & & $58(84.06)$ & $11(15.94)$ & \\
\hline \multicolumn{7}{|l|}{ Depth of invasion } \\
\hline $\mathrm{T} 1 / \mathrm{T} 2$ & $16(48.48)$ & $17(51.52)$ & 0.084 & $22(66.67)$ & $11(33.33)$ & 0.123 \\
\hline $\mathrm{T} 3 / \mathrm{T} 4$ & $119(64.32)$ & $66(35.68)$ & & $146(78.92)$ & $39(21.08)$ & \\
\hline \multicolumn{7}{|l|}{ Lymph node metastasis } \\
\hline No & $47(52.81)$ & $42(47.19)$ & 0.021 & $62(69.66)$ & $27(30.34)$ & 0.031 \\
\hline $\mathrm{N} 1-2$ & $88(68.22)$ & $41(31.78)$ & & $106(82.18)$ & $23(17.83)$ & \\
\hline \multicolumn{7}{|l|}{ Distant metastasis } \\
\hline MO & $53(63.86)$ & $30(36.14)$ & $<0.001$ & $74(69.81)$ & $32(30.19)$ & 0.013 \\
\hline M1 & $53(39.26)$ & $82(60.74)$ & & $94(83.93)$ & $18(16.07)$ & \\
\hline \multicolumn{7}{|l|}{ TNM stage } \\
\hline$|+| \mid$ & $37(49.33)$ & $38(50.67)$ & 0.006 & $52(69.33)$ & $23(30.67)$ & 0.049 \\
\hline$I I I+I V$ & $98(68.53)$ & $45(31.47)$ & & $116(81.11)$ & $27(18.89)$ & \\
\hline
\end{tabular}

that CD147 expression was correlated with tumor invasion and metastasis in triple-negative breast cancer; in human astrocytomas and meningiomas, overexpression of CD147 was found in high-grade tumors, and it was positively correlated with WHO grades [17]. What's more, CD147 expression has always been associated with a poor survival of cancer patients $[18,19]$. As for colorectal carcinoma, previous studies have got similar results. Zheng et al. [20] found that CD147 protein was up-regulated in colorectal cancer, without the alteration of its glycosylation or mRNA level. Stenzinger showed in their study that CD147 expression was associated with clinical TNM stage of CRC [21]. A recent study showed that CD147 was an independent prognostic factor for disease-free survival of CRC patients [22]. In our study, we have found that CD147 expression was correlated with lymph node metastasis, distant metastasis, as well as TNM stage for CRC; What's more, it is an independent prognostic factor for CRC patients. Our results have highlighted the important role of CD147 in the metastasis of CRC and its prognostic value in patients, both of which suggesting its clinical significance. Recently, some novel roles of CD147 in cancer have also been found. $\mathrm{Xu}$ et al. [23] uncovered a role of CD147 in transforming fibroblasts to cancer-associated fibroblasts, which in turn induced epithelial-to-mesenchymal (EMT) transition of breast cancer cells. These findings support a novel role of CD147 in regulating the interaction between cancer and stroma. Hibino et al. [24] found that the calcium-binding proteins S100A9 may serve as a novel ligand for CD147 to promote melanoma metastasis. ERK1/2 signaling pathway was found to be involved in CD147-mediated proliferation and invasion of gastric cancer cell line SGC7901 [25]. In another study, hypoxic microenvironment was postulated to be a major initiator of the overexpression of CD147 [26]. In addition, it has been reported that CD147 might be involved in drug resistance in different cancer types, via different mechanisms [22, 27]. The above discoveries suggested that CD147 can be involved in several malignant transformation processes other than inducing MMPs, and it may exert its function as a member in networks, instead 


\section{a}

Survival Functions

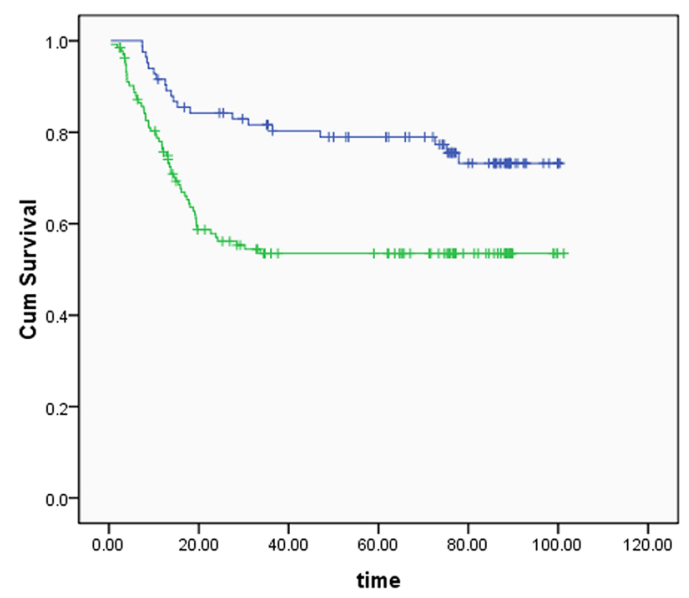

CD147

$\neg$ negative

$\neg$ positive

+ negative-censored

b

Survival Functions

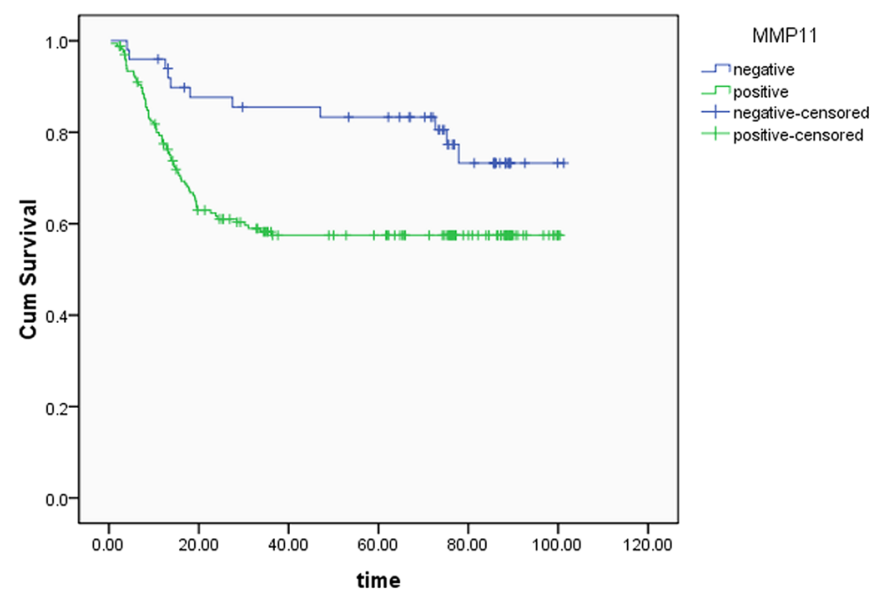

c

Survival Functions

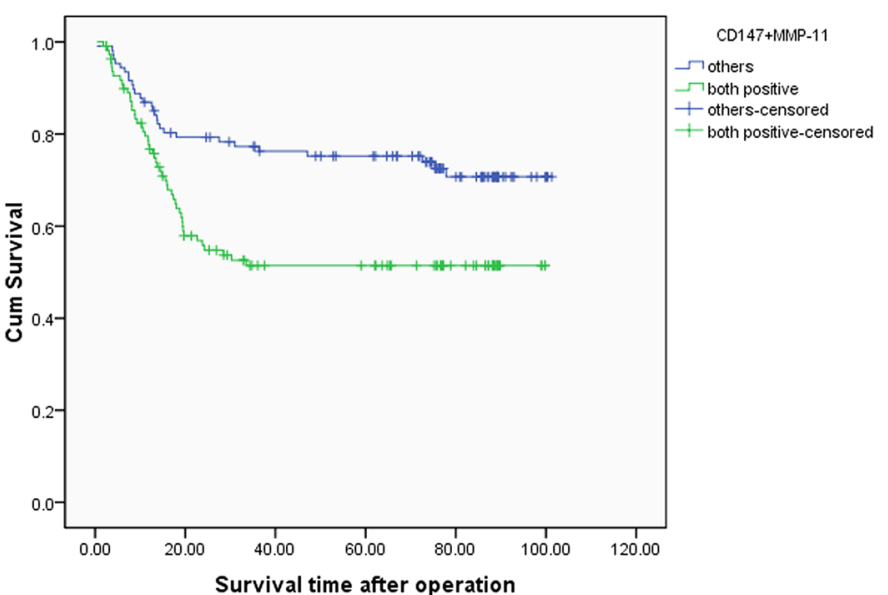

Fig. 4 Kaplan-Meier overall survival analysis of colorectal cancer patients. Survival analysis was performed according to the expression status of a CD147 $(P=0.001)$, b MMP-11 $(P=0.009)$, and c CD147 combined with MMP-11 $(P=0.002)$, respectively 
Table 4 Cox proportional hazard regression model analysis

\begin{tabular}{|c|c|c|c|c|c|c|}
\hline \multirow[t]{2}{*}{ Variables } & \multicolumn{3}{|c|}{ Univariate Cox's regression analysis } & \multicolumn{3}{|c|}{ Multivariate Cox's regression analysis } \\
\hline & Relative risk & $95 \% \mathrm{Cl}$ & $P$ value & Relative risk & $95 \% \mathrm{Cl}$ & $P$ value \\
\hline \multicolumn{7}{|l|}{ Differentiation } \\
\hline Moderate-well versus poor & 0.397 & $0.244-0.646$ & $<0.001$ & & & \\
\hline \multicolumn{7}{|l|}{ Lymphovascular invasion } \\
\hline Present versus absent & 3.606 & $2.310-5.627$ & $<0.001$ & 1.611 & $1.013-2.561$ & 0.044 \\
\hline \multicolumn{7}{|l|}{ CD147 expression } \\
\hline Positive versus negative & 2.364 & $1.422-3.931$ & 0.001 & 2.023 & $1.193-3.430$ & 0.009 \\
\hline \multicolumn{7}{|l|}{ MMP-11 expression } \\
\hline Positive versus negative & 2.281 & $1.206-4.316$ & 0.011 & 2.094 & $1.083-4.047$ & 0.028 \\
\hline \multicolumn{7}{|l|}{ Tstage } \\
\hline $\mathrm{T} 1+\mathrm{T} 2$ versus $\mathrm{T} 3+\mathrm{T} 4$ & 4.432 & $1.619-12.132$ & 0.004 & & & \\
\hline \multicolumn{7}{|l|}{ N stage } \\
\hline $\mathrm{N} 0$ versus $\mathrm{N} 1+\mathrm{N} 2+\mathrm{N} 3$ & 6.616 & $3.486-12.556$ & $<0.001$ & & & \\
\hline \multicolumn{7}{|l|}{ Metastasis } \\
\hline No versus yes & 32.746 & $12.946-82.860$ & $<0.001$ & 25.970 & $10.001-67.442$ & $<0.001$ \\
\hline
\end{tabular}

of a dominant sector, maybe that's why discrepancies exist in different studies.

As a member of the MMPs family, MMP-11 has long been postulated to play an important role in tumorigenesis, invasion, metastasis and poor clinical outcome in malignant tumors. While owing to its complex biological property, the role of MMP-11 has not been that established as other MMPs, such as MMP-2 and MMP-9. The mechanism by which MMP-11 participates in tumor progression is also unique. On the one hand, it belongs to the MMPs family, so it possesses some common properties of the family; on the other hand, it is devoid of enzymatic activity against the matrix components which is thought to be the most prominent feature of the family, so it may not have that much power in paving the path for tumor cells during their migration and invasion into the healthy tissue effectively just like other MMPs. In a study conducted by Boulay et al. [28], a new and unexpected role for MMP was revealed, that is, tumorigenesis induced by MMP-11 does not result from increased neo-angiogenesis or cancer cell proliferation, but from decreased cancer cell death through apoptosis and necrosis, indicating that the cellular function of MMP-11 is to favor cancer cell survival in the stromal environment. Similarly, MMP-11may also play a role in lobular carcinogenesis through increasing resistance to anoikis [9]. Recently a study reported the relationship between MMP11 and inflammation. MMP-11 expression by mononuclear inflammatory cells (MICs) coupled with the expression of pro-inflammatory proteins support tumor escape and invasion, hence promoting metastasis, so they claim that the expression of MMP-11 may constitute a useful biological marker for pro-metastatic MICs [29].
Interestingly, there might also be a dual role of MMP11 in cancers. It plays an oncogenic role in cervical cancer [30] and gastric cancer [31]. It can also serve as a predictor of prognosis, overexpression of MMP-11 was associated with poor prognosis in breast ductal carcinoma [8] and advanced gastric cancer [10]. In our study, it is significantly related with survival of CRC patients and can also serve as an independent prognostic factor in multivariate analysis; what's more, MMP-11 was associated with lymph node metastasis and distant metastasis, and also with tumor's TNM stage, which was similar with results in non-small cell lung cancer and breast cancer [32, 33]. However, Brasse et al. [34] found a paradoxical role of MMP-11 during the hematogenous metastatic process in mice. In their research, MMP-11 favors the onset and growth of lung metastasis but limiting lung foci number, and inhibiting the cancer cell dissemination to other organs. In another study, there was an inverse relationship between MMP-11 expression and predictors of poor prognosis of papillary thyroid carcinoma [35]. All the reported results indicated a multidimensional role of MMP 11 in cancer. If this is also the situation with other MMPs, although it's just a bold presume without enough evidence up to date, maybe the dismal curative effect of the anti-MMPs new drugs can be explained. Studies with more cases and more functional investigation are necessary.

\section{Conclusions}

Our study has demonstrated that CD147 and MMP-11 are overexpressed in colorectal cancer, and there's a positive correlation and co-localization between these two 
proteins. Both CD147 and MMP-11 might act as tumor promoters during the progression of CRC patients. What's more, the two proteins are associated with patients' survival. These indicated that CD147 and MMP11 might become potential prognostic markers as well as therapeutic targets for CRC patients.

\author{
Abbreviations \\ CRC: colorectal cancer; MMPs: matrix metalloproteinases; ECM: extracellular \\ matrix; EMMPRIN: extracellular matrix metalloproteinase inducer; TMA: tissue \\ microarray.
}

\section{Authors' contributions}

Conception and design: $\mathrm{CYH}$ and $\mathrm{XYT}$. Performing the experiments: $\mathrm{CXY}, \mathrm{XYT}$, $X Y G, Y Y Y$. Evaluation of immunohistochemistry results: $B D$ and $M Z$. Analysis and interpretation of data: $X Y T$ and $C Y H$. Writing of the manuscript: $C X Y$ and $X Y T$. All authors read and approved the final manuscript.

\section{Author details \\ ${ }^{1}$ Key Laboratory of Carcinogenesis and Translational Research, Ministry of Edu- cation/Beijing, Department of Hepato-Pancreato-Biliary Surgery, Peking Uni- versity Cancer Hospital and Institute, Beijing, People's Republic of China. 2 Department of Gastroenterological Surgery, Peking University People's Hospital, Beijing, People's Republic of China. ${ }^{3}$ Key laboratory of Carcinogen- esis and Translational Research (Ministry of Education/Beijing), Department of Biochemistry and Molecular Biology, Peking University Cancer Hospital \& Institute, Beijing, People's Republic of China.}

\section{Acknowledgements}

This study was supported by International Science \& Technology Cooperation Program of China (approval \#: 2013DFG32720), Beijing Municipal Natural Science Foundation (approval \#: 7153161), National natural science funding (approval \#: 81441071, 61372028 and 81272765), Capital characteristic clinical application research (approval \#: Z121107001012083).

\section{Competing interests}

The authors declare that they have no competing interests.

Received: 10 May 2015 Accepted: 19 October 2015

Published online: 27 October 2015

\section{References}

1. Winawer S, Fletcher R, Rex D, Bond J, Burt R, Ferrucci J, et al. Colorectal cancer screening and surveillance: clinical guidelines and rationaleupdate based on new evidence. Gastroenterology. 2003;124(2):544-60. doi:10.1053/gast.2003.50044.

2. Alberts SR, Horvath WL, Sternfeld WC, Goldberg RM, Mahoney MR, Dakhil $\mathrm{SR}$, et al. Oxaliplatin, fluorouracil, and leucovorin for patients with unresectable liver-only metastases from colorectal cancer: a North Central Cancer Treatment Group phase II study. J Clin Oncol. 2005;23(36):9243-9. doi:10.1200/jco.2005.07.740.

3. Muratore A, Zorzi D, Bouzari H, Amisano M, Massucco P, Sperti E, et al. Asymptomatic colorectal cancer with un-resectable liver metastases: immediate colorectal resection or up-front systemic chemotherapy? Ann Surg Oncol. 2007;14(2):766-70. doi:10.1245/s10434-006-9146-1.

4. Basset P, Bellocq JP, Wolf C, Stoll I, Hutin P, Limacher JM, et al. A novel metalloproteinase gene specifically expressed in stromal cells of breast carcinomas. Nature. 1990;348(6303):699-704. doi:10.1038/348699a0.

5. Pei D, Weiss SJ. Furin-dependent intracellular activation of the human stromelysin-3 zymogen. Nature. 1995;375(6528):244-7. doi:10.1038/375244a0.

6. Pei D, Majmudar G, Weiss SJ. Hydrolytic inactivation of a breast carcinoma cell-derived serpin by human stromelysin-3. J Biol Chem. 1994:269(41):25849-55

7. Manes S, Mira E, Barbacid MM, Cipres A, Fernandez-Resa P, Buesa JM, et al. Identification of insulin-like growth factor-binding protein-1 as a potential physiological substrate for human stromelysin-3. J Biol Chem. 1997:272(41):25706-12.

8. Min KW, Kim DH, Do SI, Pyo JS, Kim K, Chae SW, et al. Diagnostic and prognostic relevance of MMP-11 expression in the stromal fibroblast-like cells adjacent to invasive ductal carcinoma of the breast. Ann Surg Oncol. 2013;20(Suppl 3):S433-42. doi:10.1245/s10434-012-2734-3.

9. Takeuchi T, Adachi Y, Nagayama T, Furihata M. Matrix metalloproteinase-11 overexpressed in lobular carcinoma cells of the breast promotes anoikis resistance. Virchows Arch. 2011:459(3):291-7. doi:10.1007/ s00428-011-1125-7.

10. Yan D, Dai H, Liu JW. Serum levels of MMP-11 correlate with clinical outcome in Chinese patients with advanced gastric adenocarcinoma. BMC Cancer. 2011:11:151. doi:10.1186/1471-2407-11-151.

11. Kessenbrock K, Plaks V, Werb Z. Matrix metalloproteinases: regulators of the tumor microenvironment. Cell. 2010;141(1):52-67. doi:10.1016/j. cell.2010.03.015.

12. Guo H, Zucker S, Gordon MK, Toole BP, Biswas C. Stimulation of matrix metalloproteinase production by recombinant extracellular matrix metalloproteinase inducer from transfected Chinese hamster ovary cells. J Biol Chem. 1997;272(1):24-7.

13. Jia L, Cao J, Wei W, Wang S, Zuo Y, Zhang J. CD147 depletion down-regulates matrix metalloproteinase-11, vascular endothelial growth factor-A expression and the lymphatic metastasis potential of murine hepatocarcinoma Hca-F cells. Int J Biochem Cell Biol. 2007;39(11):2135-42. doi:10.1016/j.biocel.2007.06.007.

14. Du ZM, Hu CF, Shao Q, Huang MY, Kou CW, Zhu XF, et al. Upregulation of caveolin-1 and CD147 expression in nasopharyngeal carcinoma enhanced tumor cell migration and correlated with poor prognosis of the patients. Int J Cancer. 2009;125(8):1832-41. doi:10.1002/ijc.24531.

15. Zhong X, Li M, Nie B, Wu F, Zhang L, Wang E, et al. Overexpressions of RACK1 and CD147 associated with poor prognosis in stage T1 pulmonary adenocarcinoma. Ann Surg Oncol. 2013;20(3):1044-52. doi:10.1245/ s10434-012-2377-4.

16. Zhao S, Ma W, Zhang M, Tang D, Shi Q, Xu S, et al. High expression of CD147 and MMP-9 is correlated with poor prognosis of triple-negative breast cancer (TNBC) patients. Med Oncol. 2013;30(1):335. doi:10.1007/ s12032-012-0335-4.

17. Tsai WC, Chen Y, Huang LC, Lee HS, Ma HI, Huang SM, et al. EMMPRIN expression positively correlates with WHO grades of astrocytomas and meningiomas. J Neurooncol. 2013;. doi:10.1007/s11060-013-1184-5.

18. Reimers N, Zafrakas K, Assmann V, Egen C, Riethdorf L, Riethdorf S, et al. Expression of extracellular matrix metalloproteases inducer on micrometastatic and primary mammary carcinoma cells. Clin Cancer Res. 2004:10(10):3422-8. doi:10.1158/1078-0432.ccr-03-0610.

19. Davidson B, Goldberg I, Berner A, Kristensen GB, Reich R. EMMPRIN (extracellular matrix metalloproteinase inducer) is a novel marker of poor outcome in serous ovarian carcinoma. Clin Exp Metastasis. 2003;20(2):161-9.

20. Zheng HC, Wang W, Xu XY, Xia P, Yu M, Sugiyama T, et al. Up-regulated EMMPRIN/CD147 protein expression might play a role in colorectal carcinogenesis and its subsequent progression without an alteration of its glycosylation and mRNA level. J Cancer Res Clin Oncol. 2011;137(4):58596. doi:10.1007/s00432-010-0919-3.

21. Jin JS, Wu CY, Lin YF, Wang JY, Yu CP, Sheu LF, et al. Higher expression of epidermal growth factor receptor is associated with extracellular matrix metalloprotease inducer in colorectal adenocarcinoma: tissue microarray analysis of immunostaining score with clinicopathological parameters. Dis Markers. 2006:22(5-6):309-16.

22. Zhu S, Chu D, Zhang Y, Wang X, Gong L, Han X, et al. EMMPRIN/ CD147 expression is associated with disease-free survival of patients with colorectal cancer. Med Oncol. 2013;30(1):369. doi:10.1007/ s12032-012-0369-7.

23. Xu J, Lu Y, Qiu S, Chen ZN, Fan Z. A novel role of EMMPRIN/CD147 in transformation of quiescent fibroblasts to cancer-associated fibroblasts by breast cancer cells. Cancer Lett. 2013;335(2):380-6. doi:10.1016/j. canlet.2013.02.054.

24. Hibino T, Sakaguchi M, Miyamoto S, Yamamoto M, Motoyama A, Hosoi J, et al. S100A9 is a novel ligand of EMMPRIN that promotes melanoma metastasis. Cancer Res. 2013;73(1):172-83. doi:10.1158/0008-5472. can-11-3843.

25. Chen L, Pan Y, Gu L, Nie Z, He B, Song G, et al. ERK1/2 signalling pathway is involved in CD147-mediated gastric cancer cell line 
SGC7901 proliferation and invasion. Exp Biol Med (Maywood). 2013; doi:10.1177/1535370213493706.

26. Yang $\mathrm{H}$, Zou W, Chen B. Overexpression of CD147 in ovarian cancer is initiated by the hypoxic microenvironment. Cell Biol Int. 2013; doi:10.1002/ cbin. 10131

27. Zhou S, Liao L, Chen C, Zeng W, Liu S, Su J, et al. CD147 mediates chemoresistance in breast cancer via ABCG2 by affecting its cellular localization and dimerization. Cancer Lett. 2013;337(2):285-92. doi:10.1016/j. canlet.2013.04.025.

28. Boulay A, Masson R, Chenard MP, El Fahime M, Cassard L, Bellocq JP, et al. High cancer cell death in syngeneic tumors developed in host mice deficient for the stromelysin-3 matrix metalloproteinase. Cancer Res. 2001;61(5):2189-93

29. Eiro N, Fernandez-Garcia B, Gonzalez LO, Vizoso FJ. Cytokines related to MMP-11 expression by inflammatory cells and breast cancer metastasis. Oncoimmunology. 2013;2(5):e24010. doi:10.4161/onci.24010.

30. Vazquez-Ortiz G, Pina-Sanchez P, Vazquez K, Duenas A, Taja L, Mendoza P, et al. Overexpression of cathepsin $F$, matrix metalloproteinases 11 and 12 in cervical cancer. BMC Cancer. 2005;5:68. doi:10.1186/1471-2407-5-68.

31. Zhao ZS, Chu YQ, Ye ZY, Wang YY, Tao HQ. Overexpression of matrix metalloproteinase 11 in human gastric carcinoma and its clinicopathologic significance. Hum Pathol. 2010;41(5):686-96. doi:10.1016/j. humpath.2009.10.010
32. Delebecq TJ, Porte H, Zerimech F, Copin MC, Gouyer V, Dacquembronne $\mathrm{E}$, et al. Overexpression level of stromelysin 3 is related to the lymph node involvement in non-small cell lung cancer. Clin Cancer Res. 2000;6(3):1086-92.

33. Cheng CW, Yu JC, Wang HW, Huang CS, Shieh JC, Fu YP, et al. The clinical implications of MMP-11 and CK-20 expression in human breast cancer. Clin Chim Acta Int J Clin Chem. 2010;411(3-4):234-41. doi:10.1016/j. cca.2009.11.009.

34. Brasse D, Mathelin C, Leroux K, Chenard MP, Blaise S, Stoll I, et al. Matrix metalloproteinase 11 /stromelysin-3 exerts both activator and repressor functions during the hematogenous metastatic process in mice. Int J Cancer. 2010;127(6):1347-55. doi:10.1002/ijc.25309.

35. Ito Y, Yoshida H, Kakudo K, Nakamura Y, Kuma K, Miyauchi A. Inverse relationships between the expression of MMP-7 and MMP-11 and predictors of poor prognosis of papillary thyroid carcinoma. Pathology. 2006;38(5):421-5. doi:10.1080/00313020600922496.

\section{Submit your next manuscript to BioMed Central and take full advantage of:}

- Convenient online submission

- Thorough peer review

- No space constraints or color figure charges

- Immediate publication on acceptance

- Inclusion in PubMed, CAS, Scopus and Google Scholar

- Research which is freely available for redistribution

Submit your manuscript at

www.biomedcentral.com/submit

(O) Biomed Central 